\title{
Decay of the X-mode into two upper-hybrid plasmons in the plasma filament. Experimental modeling and theoretical description
}

\author{
L.V. Simonchik ${ }^{1}$, A.B. Altukhov ${ }^{2}$, V.I. Arkhipenko ${ }^{1}$, A.D. Gurchenko ${ }^{2}$, \\ E.Z. Gusakov ${ }^{2}$, A.Y. Popov ${ }^{2}$ and M.S. Usachonak ${ }^{1}$ \\ ${ }^{1}$ Institute of Physics of NAS of Belarus, Minsk, Belarus, 1.simonchik@ifanbel.bas-net.by \\ ${ }^{2}$ Ioffe Institute, St-Petersburg, Russia
}

The propagation of electron-cyclotron (EC) waves and their absorption in plasma until recently were believed to be well described in the framework of the linear theory and therefore predictable in detail. A theoretical analysis of such nonlinear phenomena as parametric decay instabilities (PDI) of the EC waves, performed under the assumption of monotonic plasma density profile, predicts an extremely high threshold for their occurrence (more than $1 \mathrm{GW}$ for backscattering instabilities) [1]. The reason for such high threshold of parametric instability is the large convective losses of the daughter waves from the region of parametric decay both in the direction of plasma inhomogeneity and of the magnetic field. In the recent decade, however, there has been accumulated a large number of observations in the ECR plasma heating experiments in tokamaks and stellarators that do not fit into a simple linear picture. The most distinct among them is the anomalous backscattering phenomena studied in detail in the second harmonic X-mode heating experiments at the Textor tokamak [2]. In these experiments it was shown that the radiation temperature of the observed frequency downshifted backscattering signal is thousand times larger than the electron temperature, and its amplitude is modulated at the magnetic island rotation frequency. According to [2], the highest level of anomalous backscattering is achieved when the plasma density in the island is equal to the upper hybrid density for the frequency equal to the half value of the pump frequency. Based on the latter observation the theoretical model [3] was proposed recently allowing to explain the effect of anomalous backscattering as a result of the two upperhybrid (UH) plasmons PDI possessing very low threshold due to trapping of nonlinearly excited plasmons in the vicinity of the density maximum that accompanies the island. The theory [3] also predicts substantial (up to $25 \%$ ) anomalous absorption due to this process.

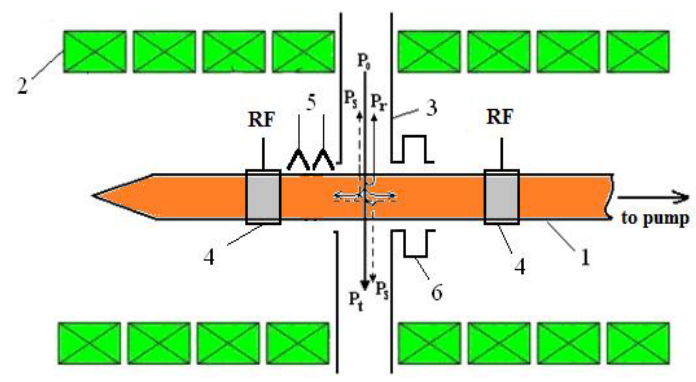

Fig. 1. Schematic of experimental setup. 1 - quartz tube; 2 - magnetic system; 3 - waveguide; 4 - RF antenna; 5 - microwave antenna; 6 - microwave cavity

In the present paper the low-threshold two-plasmon PDI leading to excitation of the trapped UH waves is modelled experimentally in the laboratory plasma. The decay occurs in a plasma filament extended in the direction of the magnetic field and produced by highfrequency discharge in long quartz tube with an inner diameter of $23.5 \mathrm{~mm}$ filled with argon at about 2 Pa pressure. Quartz tube passes through the waveguide $(72 \times 34$ $\mathrm{mm}^{2}$ ) in parallel to the wide wall (see Fig. 1). The RF power of about $100 \mathrm{~W}$ at frequency of $27 \mathrm{MHz}$ is supplied to the ring electrodes placed outside of the tube and disposed on both sides of the waveguide at a distance of $300 \mathrm{~mm}$.

At the maximal RF power the volume averaged plasma density measured using the cavity diagnostics is about $10^{10} \mathrm{~cm}^{-3}$ changing by $15-20 \%$ at the variation of the magnetic field from 0 to $450 \mathrm{Oe}$. The radial distribution of the plasma luminosity is approximated by expression $\left(1-r^{2}\right)^{1.6}$. Assuming it to be proportional to the plasma density we get the maximal density in the discharge about $2 \times 10^{10} \mathrm{~cm}^{-3}$.

The X-mode microwave pulses (up to $160 \mathrm{~W}$ ) at frequency $f_{0}=2.35 \mathrm{GHz}$, substantially higher than the EC and $\mathrm{UH}$ values, are incident onto the plasma along the waveguide. The temporal behaviors of the transmitted and reflected microwave signal, as well as the plasma luminosity are monitored in the experiment at the different pump power and magnetic field.

The theoretical model of the low-threshold twoplasmon decay proposed in [3] consists of two parts shown schematically in Fig. 2. Firstly, it is a model of the PDI linear stage describing the absolute instability leading to extra-ordinary $t_{X}$-wave decay into two $\mathrm{UH}$ plasmons $l_{\mathrm{UH}}\left(f_{1}\right)$ and $l_{\mathrm{UH}}\left(f_{2}\right)$, which are exponentially growing from the thermal noise level. Secondly, it is a model of the absolute instability nonlinear saturation as a result of cascade of several low-threshold decays in which UH waves $l_{\mathrm{UH}}$ ' trapped in plasma and low frequency waves (ion sound $l_{\mathrm{IS}}$ in the case of our experiment) are excited. The saturation level determines the efficiency of the anomalous absorption.

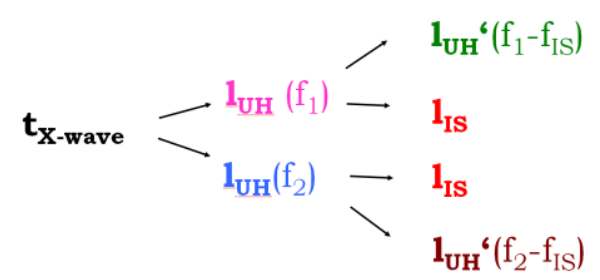

Fig. 2. The scheme of the two-plasmon decay and it's saturation

The observations of the microwave anomalous absorption at the pump frequency higher than the second electron cyclotron harmonic are performed at the power 
of $160 \mathrm{~W}$ and magnetic field smaller than $42 \mathrm{mT}$. At a small plasma density no distortions of the microwave pulse transmitted through the plasma or reflected from it are observed (Fig. 3a).
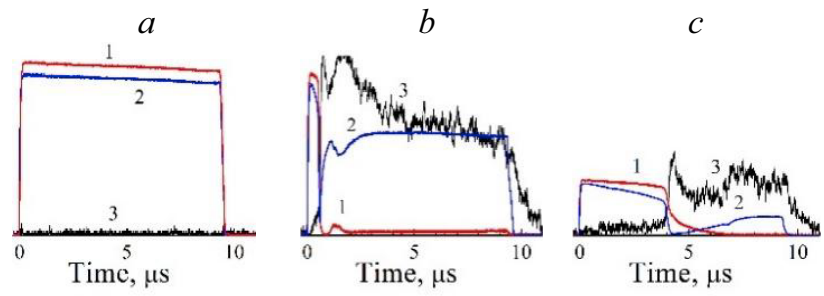

Fig. 3. Waveforms of transmitted (1), reflected (2) pulses and of the plasma luminosity (3). $a$-small plasma density, $b-P_{0}=$ $160 \mathrm{~W}, c-P_{0}=30 \mathrm{~W}$

However at plasma density exceeding a critical value a fast decrease of both transmitted and reflected power is observed indicating the turning on of the strong anomalous absorption (see Fig. 3b). This effect is accompanied by a sharp growth of the plasma luminosity also shown in this figure. The time delay of the anomalous phenomena appearance is dependent on the pump power, as it is seen in Fig. $3 b-c$.

The critical density needed for switching on of the anomalous phenomena is growing with decreasing magnetic field, as it is shown by circles in Fig. 4. As it is seen there, this dependence is close to the theoretical UHR density dependence on the magnetic field plotted in the figure for the half pump frequency

$$
n_{e}=\frac{m}{4 \pi e^{2}}\left(\pi^{2} f^{2}-\left(\frac{\theta B}{m_{e} c}\right)^{2}\right)
$$

The dependence of the two-plasmon instability growth rate on the pump power are studying as well. The measurements are performed for two magnetic fields 350 and $400 \mathrm{Oe}$, but at the constant averaged plasma density of about $10^{10} \mathrm{~cm}^{-3}$ and Ar pressure of about 1.5 Pa. The results for the inverse time of the anomalous absorption turning on shown in Fig. 5 appear to be in a qualitative agreement to the theory predictions for the growth rate increase with increasing pump power and for the PDI power threshold decrease with growing magnetic field. The threshold increases while the gas pressure decreases.

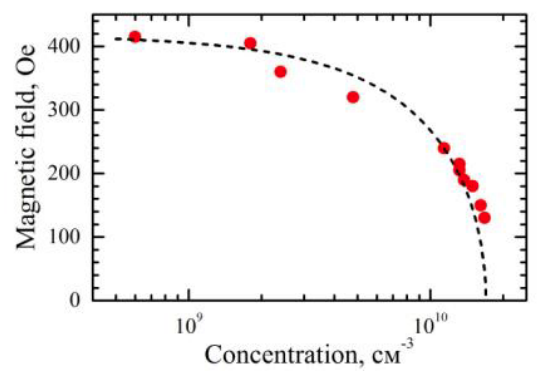

Fig. 4. Dependence of the density critical for onset of the anomalous absorption on the magnetic field. Broken line - UHR condition for $1.175 \mathrm{GHz} ; P_{0}=160 \mathrm{~W}$

Using the spatially resolved plasma luminosity measurements we study the localization of the anomalous absorption, which appears to be non-central in qualitative agreement with the theoretical expectations based on the UH eigen mode localization. The radial position of the plasma radiation drastic growth was shown to be dependent on the magnetic field.

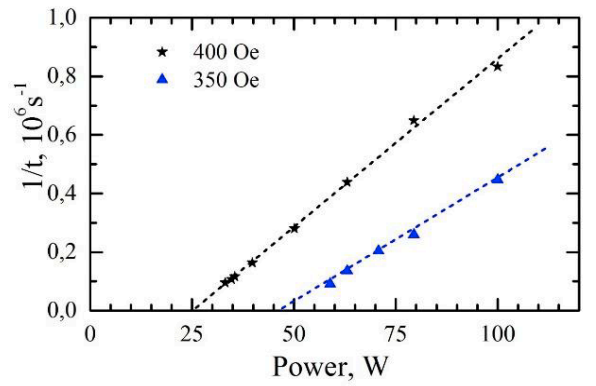

Fig. 5. Dependence of the inverse time of the anomalous absorption onset on the pump power for different magnetic fields

The low frequency waves excited in plasma as a result of PDI saturation are investigated using the UH resonance backscattering diagnostics utilizing the frequency range of 1.1-1.2 GHz. The temporal variation of the BS spectrum is demonstrated in Fig. 6. The white line on the top of the figure shows the waveform of the transmitted signal. The most intensive BS signal is observed in the period of the strongest suppression of the transmitted signal, thus in the period of the strong anomalous absorption onset. During this period the BS signal is enhanced by two orders of magnitude. The frequency shift of the BS signal is close to $2-3 \mathrm{MHz}$, which corresponds to the ion acoustic frequency range along with the PDI saturation mechanism discussed in theory.

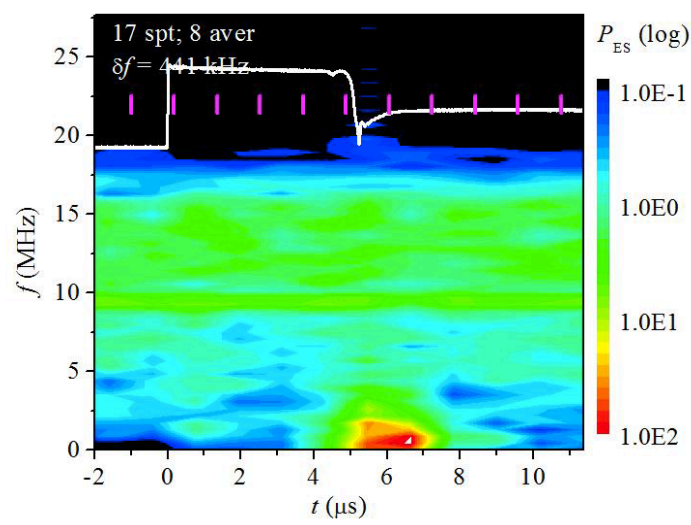

Fig. 6. The temporal evolution of the BS spectrum. The upper curve corresponds to the transmitted pulse

The work was performed under support of the BRFBR grant F16R-095 and RSF grant 16-12-10043.

\section{References}

1. Porkolab M. and Cohen B.I. // Nucl. Fusion. 1988. V. 28, No. 2. P. 239-254.

2. Nielsen S.K., Salewski M. et al. // Plasma Phys. Control. Fusion. 2013. V. 55. 115003.

3. Gusakov E.Z. and Popov A.Yu. // Physics of Plasmas. 2016. V. 23. 082503. 